\title{
Thyroid testing in acutely ill patients may be an expensive distraction
}

\author{
Lakdasa D. Premawardhana*1,2 \\ 'Section of Endocrinology, YYF Hospital, Caerphilly, UK \\ ${ }^{2}$ Centre for Endocrine and Diabetes Sciences, University Hospital of Wales, Cardiff, UK \\ ${ }^{*}$ Corresponding author: premawardhanald@cardiff.ac.uk
}

\begin{abstract}
In health, an efficient negative feedback mechanism maintains serum thyroid hormone concentrations within an exquisitely controlled narrow range. Therefore any change that occurs to thyroid hormones in intrinsic thyroid disease is concordant and easy to interpret. Optimal functioning of the many tissues they influence is thereby facilitated.

The situation in acute illnesses is different. Mechanisms that operate in these circumstances influence the hypothalamic-pituitary-thyroid axis and its components producing thyroid test results, which are discordant, do not fit recognizable patterns and are difficult to interpret. The yield of abnormalities is also low (about 7\%). As many studies indicate, thyroid tests are expensive and consume large amounts of the hospital budget and resources of hospital laboratories. Other studies have shown that when abnormalities are detected, clinicians do not intervene or follow up these subjects. Therefore the clinical utility of thyroid testing in acutely ill patients is debatable. Interventions to change requestor behaviour with regard to thyroid testing in acutely ill subjects and the success of some audit and educational interventions are worthy of note.

Thyroid testing in acutely ill patients is often an expensive distraction and is of limited clinical value. Targeted thyroid testing should be offered in this group only to those with: (a) symptoms or signs of thyroid disease e.g. goiter or orbitopathy; (b) risk factors for thyroid disease, previous or family history of thyroid disease; (c) taking drugs which potentially affect thyroid function e.g. thyroxine replacement therapy, amiodarone, lithium, mechanistic target of rapamycin (mTOR) inhibitors, interferon, alemtuzumab etc; (d) unexplained tachydysrhythmias.
\end{abstract}

Key words: thyroid tests; acute illness; guidelines

\section{Background}

The relationship between the two biologically active thyroid hormones, free thyroxine $\left(\mathrm{fT}_{4}\right)$ and free triiodothyronine $\left(\mathrm{fT}_{3}\right)$, and thyroid stimulating hormone (TSH), the pituitary hormone that controls them, is tightly preserved in many diverse physiological and pathological conditions. Blood concentrations of $\mathrm{fT}_{4}$ and $\mathrm{fT}_{3}$ are maintained within a narrow range, which remains constant throughout life with little intra-individual variability $(1,2)$. This constancy probably reflects an individual's pituitary "set point" (3). This close relationship is important because of the wide array of physiological activities that thyroid hormones influence. Both autoimmune and non-autoimmune intrinsic thyroid diseases (e.g. Graves' disease (GD), Hashimoto's thyroiditis (HT) or nodular thyroid disease) have the potential to disturb this relationship. The resulting thyroid hormone abnormalities and the new "steady state" are predictable and are easy to understand because the changes are concordant. For example, in GD there is stimulation of the TSH receptor by the thyrotropin receptor antibody (TRAb), resulting in increased blood concentrations of $\mathrm{fT}_{4}$ and $\mathrm{fT}_{3}$ (4). The finely tuned negative feedback mechanism called the hypothalamic-pituitary-thyroid axis (HPT) causes inhibition of the 
thyrotrophic hormones, suppressing TSH concentrations often into the undetectable range (5). The opposite happens in HT where decreased blood concentrations of $\mathrm{fT}_{4}$ and $\mathrm{fT}_{3}$, cause thyrotrophs to increase TSH secretion (6). Clinicians can therefore derive information about current thyroid homeostasis by measuring $\mathrm{fT}_{4}$ and $\mathrm{fT}_{3}$, the biologically active forms of these hormones currently preferred by most laboratories, and TSH in the blood.

Such thyroid hormone testing is common these days (7). The availability of technically simple, quick and relatively cheap automated analysers has increased the demand on laboratories all over the world. The United Kingdom (UK) has seen an exponential rise in the number of thyroid tests every year - 10 million thyroid tests which included fT4, fT3 and $\mathrm{TSH}$, costing an estimated 30 million sterling pounds a year $(8,9)$. The biochemistry laboratory in our hospital in Cardiff which has approximately 1000 beds and serves a population of about 500,000 did 129,953 combined $\mathrm{fT}_{4}$ and TSH tests, $15,949 \mathrm{TSH}$ tests alone, and about $4600 \mathrm{fT}_{3}$ tests in the year 2013/14. The cost for reagents alone was approximately $£ 56,000$ (about $2.35 \%$ of total reagent costs for the centre) - this does not include staff (phlebotomist, technician, biochemist and portering) and transport costs, and costs of calibrators, controls and maintenance (personal communication, Dr. C. Evans, Cardiff). In Zagreb, in a 550-bedded teaching hospital, where approximately 16,800 TSH tests, $11,400 \mathrm{fT}_{3}$ tests and $14,400 \mathrm{fT}_{4}$ tests are done annually, about $7 \%$ of the total annual laboratory budget is spent on them (personal communication, Professor AM Simundic, Zagreb).

Clinicians have contributed to an increasing laboratory workload by adopting a very low threshold for testing, in an attempt at diagnosing subtle thyroid dysfunction (10-12). The clinical features of thyroid dysfunction are non-specific and often mild, e.g. tiredness and lack of energy, and the clinician has to display a high index of suspicion to diagnose them. There is also a move towards investigating and treating subtle thyroid abnormalities in general and particularly in some circumstances e.g. pregnancy (13).

However, clinicians are also responsible for requesting thyroid tests inappropriately, particularly when individual patient circumstances do not warrant such tests (14). For example, some hospitals adopt a policy of a "minimal set" of investigations in clinical areas admitting acutely ill patients (often including thyroid tests). But others don't, and often tests are done at the sole discretion of the admitting health care professional, with scant regard for patient symptoms or presenting diagnosis. In fact it is estimated that of all requests that are made to hospital laboratories, about 25 - 40\% are unnecessary. Within biochemistry the estimate of unnecessary tests is even higher at between 26-98\% (15).

In this regard, the clinical utility of laboratory diagnostic pathways needs to be highlighted, particularly in thyroid testing (16). These pathways are helpful in differentiating between the causes of frequently presenting clinical syndromes and in investigating rare or complex syndromes, which clinicians do not see often. They are evidence based, comply with regulations and are suited to local conditions. They will also have the potential to inform the clinician of conflicting or potentially confusing pre-analytical circumstances, when used within hospital electronic data systems. These pathways therefore would have advised against thyroid testing in acutely ill patients in the setting of the Acute Medical Admissions Unit - unless specific indications or criteria were fulfilled.

Such indiscriminate thyroid testing should be discouraged in acutely ill patients for several reasons: (a) test results are often discordant and difficult to interpret (17) particularly for the generalist or nonspecialist; (b) test results are often ignored and not followed up by clinicians ordering them; and (c) they therefore do not enhance patient management or clinical outcome, but only add to the expenses associated with thyroid testing.

\section{The hypothalamic-pituitary-thyroid (HPT) axis}

There is an extremely well controlled and finely tuned negative feedback mechanism, the HPT axis (Figure 1), which maintains thyroid hormones within a very narrow range throughout life (5). The individual components of the HPT have now been 


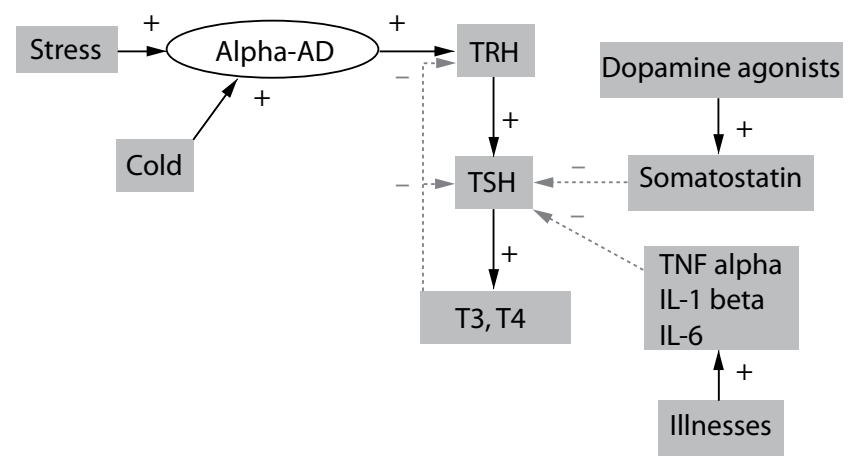

Figure 1. The hypothalamic-pituitary-thyroid axis (HPT axis). TRH is secreted by the hypothalamus, TSH by the pituitary gland and $\mathrm{T}_{4}$ and $\mathrm{T} 3$ by the thyroid gland. Both intrinsic and extrinsic factors affect the HPT axis.

$\mathrm{TRH}$ - thyrotropin releasing hormone; TSH - thyroid stimulating hormone; TNF - tumour necrosis factor; IL - Interleukin; Alpha$\mathrm{AD}-$ alpha adrenergic drive; $+=$ stimulation, $-=$ inhibition.

worked out. The hypothalamus secretes thyrotropin releasing hormone $(\mathrm{TRH})$ which is the main stimulus to pituitary TSH secretion. TRH secretion is in turn inhibited by $\mathrm{fT}_{3}$ and $\mathrm{fT}_{4}$ and influenced positively and negatively by a variety of other substances depicted (Figure 1), both in physiological and disease states $(7,8)$. Pituitary TSH secretion, which is pulsatile and is highest late evening and early morning, is inhibited also by $\mathrm{fT}_{3}$ and $\mathrm{fT}_{4}$ (18). The thyroid secretes its major product the prohormone $\mathrm{fT}_{4}$, stimulated by $\mathrm{TSH}$, which is then converted to the active hormone $\mathrm{fT}_{3}$, by intracellular deiodinase enzymes both in peripheral and central tissues (pituitary and hypothalamus) (19). The active hormone $\mathrm{fT}_{3}$, gains access to cells and their nuclear thyroid hormone receptors through the mechanism of thyroid hormone transporters such as organic anion transporter family member $1 \mathrm{C} 1$ (OATP1C1), monocarboxylate transporter 8 (MCT8) and MCT10 (20). The effects of other proteins such as cytokines, which affect the HPT axis, are depicted in Figure 1.

\section{Non thyroid illness syndrome (NTIS) - thyroid tests are discordant and difficult to interpret}

The effects of acute illness (and some chronic illnesses) on thyroid hormones and TSH, has been known since the 1970s and is currently referred to as the non-thyroidal illness syndrome (NTIS) (21). There is an ongoing debate about whether the changes of NTIS are physiological (or "adaptive") or pathological (or "maladaptive") (22). In many instances it is a combination of the two, compounded by the effects of drugs $(23,24)$. There appears to be no significant intrinsic thyroid dysfunction despite the occasional severity of thyroid hormone abnormalities in NTIS (25). However, the jury is still out about intervention, as current studies do not indicate a need for thyroid hormone replacement therapy (25).

Many mechanisms have been implicated in NTIS, and they may be multiple in any one individual $(24,26)$. Amongst the most likely are: (a) abnormal $\mathrm{TRH}$ and TSH secretion; (b) defective deiodinase activity; (c) thyroid hormone binding protein (thyroglobulin, albumin and transthyretin) and transporter (e.g. MCT8) defects; and (d) altered nuclear thyroid hormone receptor activity. Although the exact mechanisms causing these changes are unknown, cytokines such as IL-1, IL-6, and TNF-a may be responsible in some types of NTIS (18). However, a discussion of these is outside the scope of this review.

The biochemical changes of NTIS may begin within the first 24 hours of illness (27). The most common abnormality is a reduction of free T3 (and a rise in reverse $\mathrm{T} 3$ which is metabolically inactive but not measured clinically). Patients are often significantly ill with a poor prognosis when $\mathrm{fT}_{4}$ also becomes reduced. In a minority of acutely ill subjects $\mathrm{fT}_{4}$ and $\mathrm{fT}_{3}$ are elevated (5). TSH is low (in about $50 \%$ of patients) or within the reference range. However, TSH is elevated during recovery in most (26). Therefore, the combinations of $\mathrm{fT}_{4} / \mathrm{T}_{3}$ and TSH at any given time are discordant (e.g. both may be low or high, with other combinations as well), difficult to interpret and confusing particularly for the generalist.

There are several studies that describe this well (Table 1). Adlan et al. found that of the 1593 consecutive patients admitted with acute illnesses to the medical assessment unit of their hospital, 857 $(53.8 \%)$ were offered thyroid tests irrespective of 
TABLE 1. Thyroid testing in subjects with acute illness

\begin{tabular}{|c|c|c|}
\hline Reference & Thyroid abnormalities & Comments \\
\hline$(29)$ & TFTs were abnormal in $20 \%(\mathrm{~N}=635)$ & $\begin{array}{l}\text { TFTs in all acute admissions } \\
\text { NTIS in } 11.6 \% \\
\text { Intrinsic thyroid disease }{ }^{\dagger} \text { in } 8.3 \%\end{array}$ \\
\hline (31) & TFTs were abnormal in $74.3 \%(\mathrm{~N}=447)$ & $\begin{array}{l}\text { TFTs in all hospitalized elderly patients } \\
\text { NTIS in } 62.2 \% \\
\text { Intrinsic thyroid disease in } 13.4 \%\end{array}$ \\
\hline (34) & $\begin{array}{l}\text { Free T4 or TSH was abnormal in } 6 \% \text { and } 7.5 \% \\
\qquad(\mathrm{~N}=751 \text { and } 255 \text { respectively) }\end{array}$ & $\begin{array}{c}\text { Free T4 and TSH in acutely ill patients in MAU } \\
\text { NTIS }-82 \% \\
\text { Intrinsic thyroid disease }-18 \%\end{array}$ \\
\hline$(32)$ & TFTs were abnormal in $14 \%(\mathrm{~N}=185)$ & $\begin{array}{l}\text { TFTs in acute haemorrhagic stroke patients } \\
\text { NTIS in } 67 \% \\
\text { Intrinsic thyroid disease }-14 \%\end{array}$ \\
\hline$(40)$ & TFTs were abnormal in $31.8 \%(\mathrm{~N}=503)$ & $\begin{array}{l}\text { TFTs in subjects with community acquired pneumonia } \\
\text { NTIS in } 31.8 \% \\
\text { Patients with known thyroid disease were excluded from analysis }\end{array}$ \\
\hline
\end{tabular}

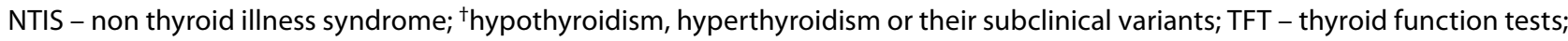
T4 - free thyroxine; TSH - thyroid stimulating hormone; MAU - medical admissions unit.

their admitting diagnosis or symptoms (28). Of the 751 subjects who finally had thyroid tests done, 84 subjects (11.2\%) had abnormal thyroid tests. But when those on thyroid replacement therapy and drugs affecting thyroid function (27 subjects) were eliminated from analysis, only 57 (7.6\%) had $\mathrm{fT}_{4}$, TSH or both, outside the reference range $-\mathrm{fT}_{3}$ is not routinely checked. $\mathrm{fT}_{4}$ was within the reference range (10.3-24.5 pmol/L) in the majority of them (69\%), below in $12.8 \%$ (probably reflecting the severity of their illness) and above the reference range in $18.2 \%$. TSH was within the reference range in $18.2 \%$, elevated in $29.1 \%$ (TSH $>4.5 \mathrm{mU} / \mathrm{L}$ ), low in $45.5 \%$ (0.01 - $0.2 \mathrm{mU} / \mathrm{L})$ and completely suppressed in $7.3 \%(<0.01 \mathrm{mU} / \mathrm{L})$ (Figure 2). When the audit was repeated four years later, 255 (21.7\%) subjects who did not have previous thyroid disease were offered thyroid tests and 19 patients (7.5\%) had abnormalities. $\mathrm{fT}_{4}$ and $\mathrm{TSH}$ were similarly discordant with no particular pattern to the abnormalities. The yield of abnormalities in those tested for thyroid dysfunction in both audits was low $(7.6 \%$ and $7.5 \%)$, the combinations were often discordant and unpredictable, and they were difficult to interpret e.g. high $\mathrm{fT}_{4}$ concentrations were associated with normal TSH and normal $\mathrm{fT}_{4}$ with low TSH concentrations (Figure 2). Small et al. from
Scotland found the "sick euthyroid state" in $11.6 \%$ of a total of 630 consecutive acute medical admissions, with $\mathrm{TSH}$ values in the reference range in all of them (29). However, $\mathrm{fT}_{4}$ and $\mathrm{fT}_{3}$ were low in $3.5 \%$ of the total group. $2.1 \%$ were given specific treatment for hypothyroidism or hyperthyroidism as thyroid tests were suggestive of intrinsic thyroid

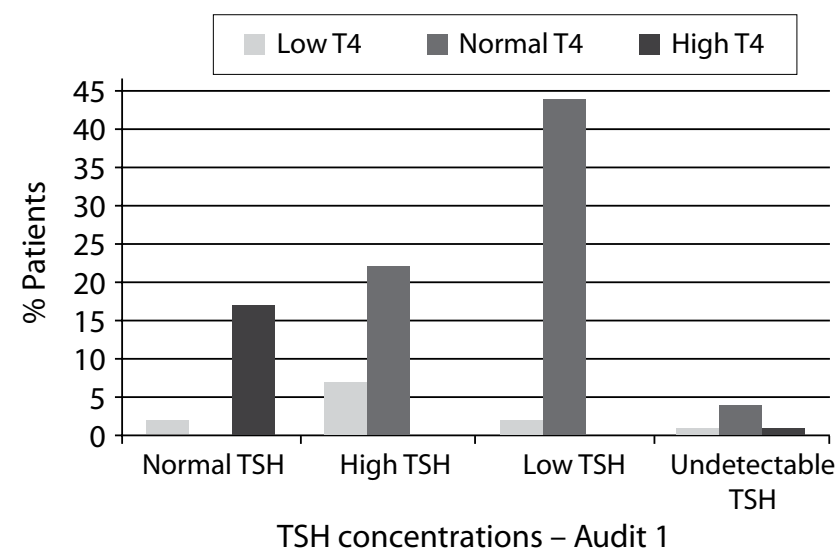

Figure 2. Pattern of thyroid abnormalities in acutely ill patients. Free thyroxine $\left(\mathrm{fT}_{4}\right)$ and $\mathrm{TSH}$ tests were discordant in many with abnormal thyroid tests and the combinations were difficult to interpret e.g. normal TSH combined with high $\mathrm{fT}_{4}$; low TSH combined normal $\mathrm{fT}_{4}$ etc. - adapted from (34).

$\mathrm{TSH}$ - thyroid stimulating hormone; $\mathrm{T} 4$ - free thyroxine 
disease. In a similar study from Italy the authors found a relatively high number of inappropriate thyroid test requests ( $22.4 \%$ of 464 requests) from the emergency department of their hospital, which were difficult to interpret and had little clinical utility (14). The yield of thyroid test abnormalities in acutely ill subjects is relatively low as the above studies indicate (between 7.4 and 20\%).

\section{NTIS occurs in non-critically ill patients}

The majority of hospital patients have non-critical illnesses. Although NTIS was initially described in critically ill patients in Intensive Therapy units (ITU), there is accumulating evidence that NTIS also occurs in subjects who are acutely but not critically ill i.e. not requiring ITU treatment (Table 2) (30-33). These studies have demonstrated a high incidence of NTIS (up to $62 \%$ in some) in subjects with acute but non-critical illness - cardiac, stroke and other illnesses such as pneumonia. Therefore, physicians should be aware of this phenomenon when thyroid tests are requested in areas where acutely ill patients are admitted even if they are not "critically" ill.

TABLE 2. Studies in non-critically ill subjects who showed biochemical features of NTIS

\begin{tabular}{|c|c|}
\hline Study (reference) & Remarks \\
\hline $\begin{array}{l}\text { Acute MI (non critical); } \\
\qquad \begin{array}{l}\mathrm{N}=331 \\
(30)\end{array}\end{array}$ & $\begin{array}{l}\text { Reverse T3 associated } \\
\text { with mortality }\end{array}$ \\
\hline $\begin{array}{l}\text { Various non critical cardiac illness; } \\
\qquad \begin{array}{c}\mathrm{N}=573 \\
(32)\end{array}\end{array}$ & $\begin{array}{c}\text { Free T3 associated with } \\
\text { survival }\end{array}$ \\
\hline $\begin{array}{l}\text { Elderly patients with various } \\
\text { non-critical illnesses; } \\
\qquad \begin{array}{c}\mathrm{N}=447 \\
\text { (31) }\end{array}\end{array}$ & $\begin{array}{l}\text { High prevalence of NTIS } \\
\qquad(62.2 \%)\end{array}$ \\
\hline $\begin{array}{l}\text { Acute stroke; } \\
\qquad \begin{array}{c}\mathrm{N}=772 \\
\text { (33) }\end{array}\end{array}$ & $\begin{array}{l}\text { Low free T3 associated } \\
\text { with short term and } \\
\text { long term outcome }\end{array}$ \\
\hline
\end{tabular}

MI - Myocardial Infarction; NTIS - Non thyroidal illness syndrome.

\section{The clinical utility of thyroid tests in the acutely ill patient}

The clinical utility of thyroid tests in acute illness remains doubtful. Clinical experience indicates that in addition to the poor yield of abnormalities and discordant and difficult to interpret thyroid hormone and TSH combinations, physicians do not intervene or follow these patients after their acute admission. In other words, these tests are not of high clinical utility.

\section{Abnormal thyroid results are ignored}

In the study by Adlan et al., $25.5 \%$ of patients with abnormal thyroid tests, the majority of whom were known to have previous thyroid disease, had either thyroid related medication introduced or dose adjusted (34). However, $74.5 \%$ of those with thyroid abnormalities had no documented intervention following the detection of thyroid abnormalities. As most of these patients were admitted under the care of "non-Endocrine specialist" physicians (specialists in Care of the Elderly Medicine, Gastroenterology etc.), it appeared that they did not have the expertise nor the inclination to deal with them and as a result there was no intervention planned - intervention defined here as planned follow up after the acute illness, or investigation and treatment while in hospital. It is clear that the majority of patients with abnormal thyroid results are not followed up.

\section{Inadequate follow up and intervention}

In the same study by Adlan et al., only $45.5 \%$ of subjects with abnormal thyroid results were followed up after the index admission (median 8 weeks; range 1-39 weeks) (34). Thyroid tests had become normal in 9 of these (36\%), but remained abnormal in 6 patients who were known to have previous thyroid disease and 10 who were not (64\%). No further action was planned for these (34). It is clear from the above that thyroid testing in this group of individuals did not influence clinical outcome. 


\section{How do we reduce thyroid testing in acutely ill patients?}

Inappropriate thyroid testing in the presence of acute illness has multiple significant consequences - unnecessary expense, increasing laboratory workload, waste of scarce resources and potential negative impact on patient health (often involving further testing and inappropriate treatment) (13). One effective way of reducing these consequences is to reduce the number of test requests by changing requestor behavior.

There are many interventions that may be employed to change requestor behaviour. Two reviews summarise them as follows: (a) educational interventions; (b) guideline and protocol development; (c) changes to funding arrangements; (d) use of existing guidelines and protocols; (e) decision making tools - request forms and computer based decision support; (f) audit and feedback (3537). Where requestor behaviour needs to be modified, the type of intervention that is relevant to local circumstances needs to be utilised.

Successful interventions to reduce thyroid testing in acutely ill subjects involving requestor education and audit, adopted in two hospitals in South East Wales, will now be described.

\section{Educational intervention}

In a 500 bedded district general hospital in Wales, 950 acutely ill patients were admitted on average per month to the medical admissions unit (MAU) this did not include patients with suspected poisoning who were admitted to a specialist ward. Each month 309 thyroid function tests, 481 lipid profiles and 818 coagulation screens were requested for these patients on average. These requests were made primarily at the time of triage by nursing staff. Following the identification that these test requests were excessive, discussions were held between the Laboratory staff and the clinicians in the MAU. They removed these three sets of investigations from the standard MAU biochemistry test request forms and agreed a new protocol for biochemical testing. They also implemented an educational intervention in the MAU as follows: (a) lectures and tutorials delivered to all clinical staff; (b) posters describing new protocol for laboratory requests were displayed in prominent areas in the MAU; (c) protocol and data presented to the Medical Directorate Governance and Safety committee for review. When pre-intervention request behaviour was compared to post-intervention request behaviour calendar month to calendar month (to rule out seasonal variation), a significant reduction was observed in thyroid tests requested per patient $(0.32$ before intervention (SD 0.05$)$ vs. 0.08 post intervention (SD 0.01); $\mathrm{P}<0.001$ ) (38). This was also true for the other tests removed from the standard request protocol. The authors of this study concluded that in addition to cost savings, this study improved the appropriateness of testing and improved patient care (38).

\section{Thyroid testing guidelines and audit}

In the study discussed above, following an initial audit, the authors issued guidelines about thyroid testing in acute illness, and repeated the audit 4 years later (34). Whereas thyroid tests were offered to $53.8 \%$ acutely ill subjects in the first audit, this was reduced to $21.7 \%$ in the second $(P=0.01)$. Furthermore as mentioned above, physicians acted upon reviewing the results and follow up was arranged for a significantly higher percentage of subjects following the second audit (Table 3 ).

TABLE 3. Comparison of thyroid testing in acutely ill subjects after implementing thyroid testing guidelines.

\begin{tabular}{lcc}
\hline & Audit 1 & Audit 2 \\
\hline Number of admissions studied & 1593 & 1176 \\
\hline Number of offered TFT, N (\%) & $857(53.8)$ & $255(21.7)^{*}$ \\
\hline $\begin{array}{l}\text { Prevalence of thyroid } \\
\text { abnormalities (\%) }\end{array}$ & 7.6 & 7.5 \\
\hline Action taken (\%) & 25.5 & $92.7^{*}$ \\
\hline Follow up (\%) & 45.5 & $85.7^{*}$ \\
\hline
\end{tabular}

*There was a significant decrease in the number of thyroid tests offered, and an increase in the proportion of patients on whom action was taken and follow up arranged, after implementing guidelines for thyroid testing in acutely ill subjects (34).

TFT - thyroid function tests. 


\section{Conclusions}

Measuring thyroid hormones and TSH in acutely ill patients is often an expensive distraction and is only of limited clinical value. The yield of abnormal thyroid tests is low, the tests are discordant and often difficult to interpret for the generalist (as they do not fit recognizable patterns), and are often ignored by the requesting physician (34). In addition to being a distraction clinically, they are also expensive to do and in some hospitals account for a significant proportion of the laboratory services budget. Education of nurses, junior doctors and senior physicians in the futility of thyroid testing during acute illness, helps in reducing the number of tests done, as has been shown in two recent studies $(34,38)$. Efforts should be made to devise a sensible, logical and easily understood set of guidelines for targeted thyroid testing in acutely ill patients in hospital medical assessment units (39).

The following criteria for targeted thyroid testing were adopted in our medical assessment unit with improved results i.e. with a significant reduction in

\section{References}

1. Fliers E, Kalsbeek A, Boelen A. Beyond the fixed setpoint of the hypothalamo-pituitary-thyroid axis. Eur J Endocrinol 2014;171:197-208. https://doi.org/10.1530/EJE-14-0285

2. Andersen S, Pedersen KM, Bruun NH. Narrow individual variations in serum $T(4)$ and $T(3)$ in normal subjects: a clue to the understanding of subclinical thyroid disease. J Clin Endocrinol Metab 2002;87:1068-72. https://doi.org/10.1210/ jcem.87.3.8165

3. Duntas LH. New insights into the hypothalamic-pituitarythyroid axis. Acta Endocrinol 2016;12:125-129. https://doi. org/10.4183/aeb.2016.125

4. Bahn RS. Autoimmunity and Graves' disease. Clin Pharmacol Ther 2012;91:577-579. https://doi.org/10.1038/ clpt.2012.10

5. Ortiga-Carvalho TM, Chiamolera MI, Pazos-Moura CC, Wondisford FE. Hypothalamus-Pituitary-Thyroid Axis. Comprhen Physiol 2016;6:1387-1428. https://doi.org/10.1002/ cphy.c150027

6. Ajjan RA, Weetman AP. The Pathogenesis of Hashimoto's Thyroiditis: Further Developments in our Understanding. Horm Metab Res 2015;4:702-710. https://doi. org/10.1055/s-0035-1548832 thyroid test requests, a higher proportion of results being acted upon, and more patients being followed up after the acute illness:

- patients with symptoms or signs of thyroid disease e.g. goiter, orbitopathy

- patients with risk factors for thyroid disease, previous or family history of thyroid disease

- patients taking drugs which potentially affect thyroid function e.g. thyroxine replacement therapy, amiodarone, lithium, mechanistic target of rapamycin (mTOR) inhibitors, interferon, alemtuzumab etc.

- unexplained tachydysrhythmias.

Changing requestor behaviour in reducing inappropriate thyroid testing in acutely ill patients is a worthwhile exercise, and emerging evidence indicates audit and education of healthcare professionals are interventions that should be explored.

\section{Potential conflict of interest}

None declared.

7. Plebani M. Errors in Clinical laboratories or errors in laboratory medicine? Clin Chem Lab Med 2006;44;750-759. https://doi.org/10.1515/CCLM.2006.123

8. Beckett GJ, Toft AD. First line thyroid function tests - TSH alone is not enough. Clin Endocrinol (Oxf) 2003;58:20-21. https://doi.org/10.1046/j.1365-2265.2003.01690.x

9. Association for Clinical Biochemistry, British Thyroid Association, British Thyroid Foundation. UK Guidelines for the use of thyroid function tests. Available at: http://www.btfthyroid.org/images/stories/pdf/tft_guideline_final_version_july_2006.pdf. Accessed June 16th 2016.

10. Bayram C, Valenti L, Britt $H$. Orders for thyroid function tests- changes over 10 years. Aust Fam Physician 2012;41:555-9.

11. Leese GP, Flynn RV, Jung RT, Macdonald TM, Murphy MJ, Morris $A D$. Increasing prevalence and Incidence of thyroid disease in Tayside, Scotland: the Thyroid Epidemiology Audit and Research Study (TEARS). Clin Endocrinol (Oxf) 2008;68:311-6.

12. Newchurch Ltd., National Pathology Benchmarking Report 2000/2001. London: Newchurch 2002. 
13. Taylor PN, Iqbal A, Minassian C, Sayers A, Draman MS, Greenwood $R$, et al. Falling Threshold for Treatment of Borderline Elevated Thyrotropin Levels-Balancing Benefits and Risks: Evidence From a Large Community-Based Study. JAMA Intern Med 2014;174:32-39. https://doi.org/10.1001/jamainternmed.2013.11312

14. Giacomini A, Chiesa M, Carraro P. Urgent thyroid stimulating hormone testing in emergency medicine: a useful tool? J Emerg Med 2015;49:481-487. https://doi.org/10.1016/j.jemermed.2015.05.003

15. Gopal Rao G, Crook M, Tillyer MI. Pathology tests: is the time for demand management ripe at last? J Clin Pathol 2003;56:243-8. https://doi.org/10.1136/jcp.56.4.243

16. Hoffmann $G$, Aufenanger J, Födinger $M$, Cadamuro J, von Eckardstein A, Kaeslin-Meyer M, Hofmann M. Benefits and limitations of laboratory diagnostic pathways. Diagnosis 2014;1:269-27. https://doi.org/10.1515/dx-2014-0045

17. Koulouri O, Moran C, Halsall D, Chatterjee K, Gurnell $M$. Pitfalls in the measurement and interpretation of thyroid function tests. Best Pract Res Clin Endocrinol Metab 2013;30:745-762. https://doi.org/10.1016/j. beem.2013.10.003

18. Fliers $E$, Kalsbeek A, Boelen A. Mechanisms in endocrinology: Beyond the fixed set point of the hypothalamus-pituitary-thyroid axis. Eur J Endocrinol 2014;171:R197-207. https://doi.org/10.1530/EJE-14-0285

19. Bianco AC, Kim BW. Deiodinases; implications of the local control of thyroid hormone action. J Clin Invest 2006;116:2571-9. https://doi.org/10.1172/JCI29812

20. Visser WE, Friesma EC, Visser TJ. Minireview; thyroid hormone transporters: the knowns and unknowns. Mol Endocrinol 2011;25:1-14. https://doi.org/10.1210/me.2010-0095

21. de Vries EM, Fliers $E$, Boelen. The molecular basis of the nonthyroidal illness syndrome. J Endocrinol. 2015;225:R67-81. https://doi.org/10.1530/JOE-15-0133

22. Fliers $E$, Bianco AC, Langouche $L$, Boelen A. Thyroid function in critically ill patients. Lancet Diabetes Endocrinol 2015;3:816-25. https://doi.org/10.1016/S22138587(15)00225-9.

23. Haugen BR. Drugs that suppress TSH or cause central hypothyroidism. Best Pract Res Clin Endocrinol Metab 2009;23:793-800. https://doi.org/10.1016/j. beem.2009.08.003

24. van den Berghe G. Non-Thyroidal Illness in the ICU: A Syndrome with Different Faces. Thyroid 2014;24:1456-65. https://doi.org/10.1089/thy.2014.0201

25. Pappa TA, Vagenakis AG, Alevizaki M. The nonthyroidal illness syndrome in the non-critically ill patient. Eur J Clin Invest 2011;41:212-20. https://doi.org/10.1111/j.13652362.2010.02395.x

26. Warner MH, Beckett GJ. Mechanisms behind the non-thyroidal illness syndrome; an update. J Endocrinol 2010;205:113. https://doi.org/10.1677/JOE-09-0412

27. Wajner SM, Maia AL. New insights toward the acute nonthyroidal illness syndrome. Front Endocrinol 2012;3:1-7. https://doi.org/10.3389/fendo.2012.00008
28. Adlan MA, Neel V, Lakra SS, Bondugulapati LNR, Pre mawardhana LD. Targeted thyroid testing in acute illness: Achieving success through audit. J Endocrinol Invest 2011;34:e210-3.

29. Small M, Buchanan L, Evans R. Value of screening thyroid function in acute medical admissions to hospital. Clin Endocrinol (Oxf) 1990;32:185-91. https://doi. org/10.1111/j.1365-2265.1990.tb00854.x

30. lervasi $G$, Pinitore A, Landi P, Raciti M, Ripoli A et al. Low T3 syndrome; a strong prognostic predictor of death in patients with heart disease. Circulation 2003;107:708-13. https://doi.org/10.1161/01.CIR.0000048124.64204.3F

31. Iglesias P, Munoz A, Prado F, Guerrero MT, Macias MC et al. Alterations in thyroid function tests in aged hospitalized patients; prevalence, aetiology and clinical outcome. Clin Endocrinol (Oxf) 2009;70:961-7. https://doi.org/10.1111/ j.1365-2265.2008.03421.x

32. Pande A, Goel VK, Rastogi A, Gupta A. Thyroid dysfunction in patients with haemorrhagic stroke. Thyr Res Pract 2016;13:19-24. https://doi.org/10.4103/0973-0354.159531

33. Alevizaki M, Synetou M, Xynos K, Pappa T, Vemmos KN. Low triiodothyronine; a strong predictor of outcome in acute stroke patients. Eur J Clin Invest 2007;37:651-7. https://doi. org/10.1111/j.1365-2362.2007.01839.x

34. Adlan MA, Neel V, Lakra SS, Bondugulapati LNR, Premawardhana LD. Targeted thyroid testing in acute illness: Achieving success through audit. J Endocrinol Invest 2011;34:210213.

35. Solomon $D H$, Hashimoto $H$, Daltroy $D$ et al. Techniques to improve clinicians' use of diagnostic tests; a conceptual framework. JAMA 1998;280:2020-24. https://doi.org/10.1001/ jama.280.23.2020

36. Oxman AD, Thomson MA, Davis DA, Haynes RB. No magic bullet; a systematic review of 102 interventions to improve professional practice. CMAJ 1995;153:1423-31.

37. Zhelev Z, Abbott R, Rogers M, Fleming S, Patterson A, Hamilton $W T$ et al. Effectiveness of interventions to reduce ordering of thyroid function tests: a systematic review. BMJ Open 2016;6:e01006. https://doi.org/10.1136/bmjopen-2015-010065

38. Willis $E$, Datta DN. Effect of an educational intervention on requesting behavior by a medical admission unit. Ann Clin Biochem 2013;50:166-8. https://doi.org/10.1258/ acb.2012.012100

39. Bellew SD, Moman R, Lohse CM, Hess EP, Bellolio MF. Validation of a decision rule for selective TSH screening in atrial fibrillation. West J Emerg Med 2015;16:195-202. https://doi. org/10.5811/westjem.2014.11.23490

40. Liu J, Wu X, Lu F, Zhao L, Shi L, Xu F. Low T3 syndrome is a strong predictor of poor outcome in patients with community acquired pneumonia. Sci Rep 2016;6:22271. https://doi. org/10.1038/srep22271 